\title{
Ontogénesis del sentido de justicia. El criterio de igualdad analizado desde la psicología del \\ desarrollo
}

\author{
Ontogeny of the sense of justice. The criterion of \\ equality analyzed by developmental psychology
}

\author{
Ontogênese do senso de justiça. 0 critério da \\ igualdade analisado a partir da psicologia do \\ desenvolvimento
}

Rodrigo Sebastián Braicovich ORCID ID: 0000-0003-2785-7293

CONICET, Argentina

Autor referente: rbraicovich@gmail.com

Historia editorial

Recibido: 13/02/2018

Aceptado: 24/06/2019

\section{RESUMEN}

El objetivo del artículo es realizar un relevamiento de las investigaciones llevadas a cabo en el ámbito de la psicología del desarrollo en relación con la ontogénesis de la tendencia a recurrir en forma espontánea e inconsciente al criterio de igualdad en las evaluaciones de escenarios intersubjetivos. Como mostraré a lo largo del trabajo, existen dos modelos marcadamente diferentes (e incluso conflictivos en ciertos aspectos) para explicar dicho surgimiento. Un primer modelo (al que llamaré "modelo dual") propone interpretar la ontogénesis del sentido de justicia en base a dos momentos diferentes, claramente secuenciados, y expresión de mecanismos psicológicos distintos y relativamente independientes: el momento de manifestación de la Aversión a la Desigualdad Desventajosa [ADD] (alrededor de los cuatro años), y el de la Aversión a la Desigualdad Ventajosa [ADV] (alrededor de los ocho años). El segundo modelo (al que llamaré "modelo unitario") propone, por el contrario, que los niños comienzan a recurrir a criterios de igualdad cerca de los 12 meses de edad, $y$, fundamentalmente, que dicho criterio 
de igualdad es independiente de -o, al menos, lógicamente previo a- la distinción entre ADD y ADV. Sugeriré, no obstante, que la divergencia entre ambos modelos no es obstáculo para concluir que los resultados de las investigaciones realizadas durante la última década ofrecen fundamentos sólidos para comenzar a comprender la especificidad de la ontogénesis de nuestro sentido de justicia.

Palabras clave: Sentido de justicia; criterios de justicia; igualdad; psicología del desarrollo

\section{ABSTRACT}

The aim of the article is to offer a review of the research in developmental psychology concerning the first manifestations of our tendency to resort -in a spontaneous and unconscious manner- to the criterion of equality in our assessment of intersubjective situations. As I will show throughout the paper, two different (and even conflicting) models have been proposed to explain those early manifestations. The first model, (which I will call "dual model") suggests that we interpret the ontogenesis of our sense of justice through two distinct and clearly sequenced moments, which are the expression of two distinct and relatively independent psychological modules: the moments of manifestation of our
Disadvantageous Inequality Aversion [DIA] (around four years of old) and our Advantageous Inequality Aversion [AIA] (around eight years old). The second model (which I will call "unitary model") suggests, on the contrary, that children begin to resort to the criterion of equality when they are around 12 months old, and that such criterion is independent from -or, at least, logically prior to- the distinction between DIA and AIA. I will suggest, nevertheless, that the differences between both models should not hinder us from concluding that the research produced throughout the last decade has provided us with a solid ground to begin to understand the specificity of the ontogeny of our sense of justice.

Keywords: Sense of justice; criteria of justice; equality; developmental psychology

\section{RESUMO}

O objetivo do artigo será fazer uma pesquisa das investigações do campo da psicologia do desenvolvimento em relação ao surgimento da tendência de se recorrer espontaneamente e inconscientemente ao critério de igualdade em nossas avaliações de cenários intersubjetivos. Embora, como vou mostrar durante todo o trabalho, há dois modelos diferentes para explicar esse surgimento, os resultados das pesquisas realizadas durante a última década oferecem bases sólidas para começar a entender a especificidade da ontogênese do nosso senso de justiça.

Palavras-chave: Senso de justiça; critério de justiça; igualdade; psicologia do desenvolvimento 


\section{$\mathrm{T}$} al como sucede cuando intentamos comprender la filogénesis de nuestro sentido de justicia (analizando la evidencia proveniente, por ejemplo, de la primatología), toda especulación acerca de su ontogénesis encuentra su mayor obstáculo en el hecho de que las investigaciones realizadas en el ámbito de la psicología del desarrollo que pueden ayudarnos a abordar estos interrogantes son sumamente recientes (como veremos, en efecto, los primeros estudios sistemáticos sobre el criterio de igualdad comienzan alrededor del año 2008), y se encuentran todavía en estado incipiente: los diseños experimentales no han sido aún homogeneizados y el repertorio conceptual utilizado en los distintos estudios está lejos de ser sistemático. El panorama, sin embargo, es bastante más alentador que en el caso de la primatología, no solo porque los estudios realizados hasta el momento permiten entrever ciertas líneas generales de consenso, sino, fundamentalmente, porque parece posible construir un modelo de interpretación sencillo que incorpore los resultados obtenidos hasta el momento.

\section{El criterio de igualdad}

Imaginemos un escenario como el siguiente: un maestro reparte entre sus alumnos una cantidad determinada de golosinas; la cantidad de alumnos es impar y la cantidad de golosinas es par. ¿En qué momento de su desarrollo comenzará el niño a evaluar dicha situación como injusta si el reparto lo ha perjudicado? ¿En qué momento lo hará si, por el contrario, se ha visto beneficiado por el reparto desigual? ¿Y cuándo comenzará a evaluar como injusto un reparto desigual que no lo afecte directamente? En este último caso, ¿qué estará dispuesto a hacer a fin de expresar su disconformidad con la situación? En su manifestación más simple y despojada, estos interrogantes atañen al surgimiento no solo del criterio de igualdad, sino también de la expectativa de igualdad, que, como veremos más adelante, lo precede necesariamente desde el punto de vista de la ontogénesis. El elemento fundamental 
para comprender la especificidad del criterio de igualdad reside en el hecho de que, sin importar la complejidad del escenario en cuestión (es decir, ya sea que se trate del reclamo de una distribución igualitaria de caramelos o el reclamo de que todos los niños en edad escolar tengan acceso gratuito a un lugar en el sistema educativo estatal), dicho criterio deja de lado por completo el desempeño previo del sujeto en cuestión, y se concentra únicamente en su pertenencia a un determinado conjunto ("No merezco tener un asiento garantizado durante el período correspondiente a $1^{\circ}$ grado de la escuela inicial por el hecho de haberme desempeñado exitosamente durante los años anteriores, sino solamente por mi edad"; "No merezco que se me permita votar en las elecciones nacionales porque he demostrado ser un ciudadano activo y comprometido, sino solamente por el hecho de ser uruguayo, argentino, húngaro, etc.”). Ahora bien: ¿cuál es el conjunto más elemental al que podemos pertenecer? Lo primero que nos viene a la mente es probablemente la mera pertenencia al género humano. Sin embargo, no sería descabellado pensar en una situación en la cual un niño siente que ha recibido un trato injusto si al perro de la familia le han dado de comer un pedazo de carne y él se ha tenido que conformar con una zanahoria, o si el gato posee una cama más abrigada que la suya. Lo que esto demuestra es simplemente que el criterio de igualdad es un criterio puramente formaly vacío de contenido, y que se agota en la exigencia de que todos los individuos que componen un determinado conjunto reciban un trato igual. Si bien el modelo de L. Kohlberg (2003) identificaba esta característica como un elemento que develaba el carácter primitivo del concepto de igualdad, es precisamente este carácter estrictamente formal el que permite comprender el hecho de que el criterio de igualdad trascienda toda barrera cultural y aún, quizás, las barreras de nuestra propia especie. La pregunta que me interesa abordar, entonces, es la siguiente: ¿en qué momento de nuestro desarrollo como individuos comenzamos a recurrir al criterio de la igualdad al momento de evaluar el entorno que nos rodea? ¿Cuándo comenzamos a exigir, o al 
menos esperar pasivamente, un trato igualitario respecto de los otros individuos que comparten el conjunto de turno?

Los estudios realizados durante la última década en el ámbito de la psicología del desarrollo pueden ser agrupados, en líneas generales, en dos modelos interpretativos: el primero de ellos (al que llamaré modelo dual) sugiere que a) a partir de los cuatro años de edad, los niños evidencian una Aversión a la Desigualdad Desventajosa [ADD], b) que recién a los ocho años de edad evidencian su contraparte, a saber, la Aversión a la Desigualdad Ventajosa [ADV], y que c) dichas tendencias son expresión de mecanismos psicológicos distintos y relativamente independientes. El segundo modelo (al que denominaré modelo unitario) plantea a) que los niños comienzan a recurrir a criterios de igualdad cerca de los 12 meses de edad, b) que dicho criterio de igualdad es independiente de -o lógicamente previo a- la distinción entre ADD y ADV, y c) que existen variables externas al criterio de igualdad que explican la brecha conocimiento-acción ("sé que debo actuar de manera igualitaria pero, a pesar de ello, no lo hago"). Como veremos, los resultados en los que se apoyan ambos modelos parecen diferir considerablemente, así como las proyecciones que se derivan de cada uno de ellos para nuestra comprensión de la ontogénesis del sentido de justicia.

\section{El modelo dual de explicación de la Aversión a la Desigualdad}

\section{Aversión a la Desigualdad Desventajosa: cuando la desigualdad nos perjudica}

Uno de los dos modelos principales que han surgido para investigar y explicar el surgimiento del criterio de igualdad en los niños ha sido desarrollado a lo largo de la última década por el grupo de investigación liderado por Peter Blake y Katharine McAuliffe. A lo largo de una serie importante de trabajos (Blake \& McAuliffe, 2011; McAuliffe, Blake, Kim, Wrangham, \& Warneken, 2013, entre otros), los autores intentaron identificar, en una serie de experimentos inspirados en la teoría de juegos 
económicos, la modalidad y el momento aproximado en el cual el criterio de igualdad se hace presente en los niños en situaciones vinculadas con la distribución de recursos. En el primero de dichos estudios (Blake \& McAuliffe, 2011), los autores diseñaron una variante del Juego del Ultimátum, a la cual denominaron "Juego de la Desigualdad" (Inequity Game), cuya dinámica era la siguiente: dos participantes (uno activo y otro pasivo) se sientan frente a un mecanismo (diseñado específicamente para el experimento) y cada uno de ellos recibe una cantidad distinta de caramelos. Luego de observar las cantidades asignadas, el participante activo tiene dos opciones: o bien activa la palanca verde, en cuyo caso ambos reciben lo que les ha sido asignado, o bien activa la palanca roja, en cuyo caso ambas bandejas se vuelcan hacia el centro y ninguno de los dos participantes recibe nada.

El diseño del experimento permite, a diferencia de otros juegos económicos clásicos, anular las variables de la reciprocidad (dado que cada participante interviene una sola vez, en lugar de realizar rondas sucesivas), y de la reputación (dado que es el experimentador quien propone en forma explícita la distribución desigual, garantizando que el eventual rechazo de una oferta desigual no opere como un castigo al otro participante por haber realizado una propuesta de ese tipo ni una advertencia a futuro). Estas dos características hacen que, a diferencia de lo que sucede en otros juegos económicos, cualquier decisión por parte del participante activo de rechazar la oferta propuesta sea, desde el punto de vista de la racionalidad utilitaria o económica, absolutamente absurda y difícil de comprender: recibir un caramelo es siempre recibir menos que cero caramelos, y el hecho de que el otro participante reciba uno o cinco caramelos no debería influir en lo más mínimo en la decisión del participante activo, dado que la recompensa del otro no afecta la suya.

A pesar de ello, el análisis final del estudio arrojó resultados sorprendentes: en el grupo de los participantes de cuatro años, el índice de rechazo de las ofertas desiguales fue apenas menor al $80 \%$ — es decir: aproximadamente ocho de cada diez 
niños prefirió no recibir nada antes que recibir algo, si el otro participante iba a recibir más que él. Más curioso aún es el hecho de que esta tendencia, difícil de explicar por sí misma, se acentuó en forma sostenida a medida que la edad de los participantes aumentaba, hasta llegar a índices de rechazo cercanos al $90 \%$ en el grupo de los participantes de ocho años. El hecho de que esta tendencia no desaparezca durante la adultez, aun en escenarios en donde no parece haber ningún tipo de razón que explique en forma plausible el rechazo de una oferta desventajosa, permite, por otra parte, rechazar de plano la idea de que los resultados de Blake \& McAuliffe puedan ser explicados apelando al escaso desarrollo intelectual de los niños de cuatro años, o a mecanismos emocionales o afectivos propios de esa etapa.

Los resultados presentados por el estudio abren interrogantes sumamente interesantes: dado que el diseño del juego descarta la dinámica de la reciprocidad, y dado que rechazar la oferta no puede beneficiar en modo alguno al que la rechaza, ¿cuál es el objetivo del rechazo? ¿A quién se dirige? ¿Es consciente el participante activo de la irracionalidad de su decisión? La fuerza de estos interrogantes se vio confirmada en un estudio posterior (McAuliffe et al., 2013) que introdujo dos variaciones fundamentales en el diseño del Juego de la Desigualdad, y permitió obtener resultados sumamente significativos: la primera variación consistió en incorporar al experimento una situación en la cual el participante activo no se veía enfrentado a otro niño, sino a un lugar vacío. El objetivo de este cambio consistía en determinar si la ADD representa un fenómeno estrictamente social o no: si lo fuera, el índice de rechazos en las situaciones en las que en niño se enfrentaba a un lugar vacío, debería ser cero. Pero no lo fue: $25 \%$ de los integrantes del grupo de cuatro años rechazó la oferta (y prefirió, por lo tanto, quedarse con nada) por el solo hecho de que una silla vacía había recibido más que ellos, y un 50\% de los integrantes del grupo de cinco años hizo lo mismo. La pregunta es nuevamente inevitable: ¿qué justificaría que el niño rechazara una oferta, por magra que sea, si la oferta mayor ni siquiera va a 
beneficiar a otro, sino a un espacio vacío en la mesa? ¿Qué procesos psicológicos podrían estar detrás de una reacción tan aparentemente irracional?

La segunda modificación introducida en el experimento consistió en alternar entre situaciones del juego en las cuales la distribución desigual era visiblemente decidida por el experimentador, y otras en las cuales la oferta era determinada por una carta tomada al azar de un mazo de cartas. Los resultados fueron nuevamente llamativos, dado que no se registraron diferencias significativas en el rechazo de ofertas injustas si la distribución injusta era producto de un sujeto o de un mecanismo azaroso impersonal. En otras palabras: los niños rechazaban las ofertas que los perjudicaban sin importar que la misma hubiera sido el producto del azar o de la decisión de una persona. Pero, si este es el caso, ¿a quién se dirige, en la mente del niño, el rechazo de la oferta injusta? Si descartamos que se dirija al otro niño, y si descartamos, con este segundo experimento, que se dirija al experimentador (en las ocasiones en que el origen de la desigualdad es producto del azar), ¿qué nos queda? ¿Dios? ¿Zeus? ¿Es posible que el niño esté asumiendo que detrás del reparto desigual se halla una mano divina que está empecinada en perjudicarlo y su rechazo representa una especie de declaración de principios ante el orden cósmico? Por extrema que parezca, no podemos descartar de plano esta explicación. Pero tampoco la alternativa opuesta, a saber: que los rechazos de las ofertas injustas no se hallan en realidad dirigidos a nadie, sino que son el resultado de un módulo psicológico especializado que se activa en forma automática, inconsciente, sin involucrar representación alguna respecto del objetivo de la acción. Desde esta perspectiva, cuando el niño rechaza una oferta desventajosa, no lo hace para castigar al otro participante, ni lo hace para protestar ante Dios o Vishnu, ni tampoco para dejar sentada su posición de desagrado frente a la injusticia que ha sufrido. Simplemente lo hace.

Ahora bien: ¿de qué tipo de mecanismo podría tratarse? ¿Cuál es su origen y cómo pudo haber evolucionado? Los autores plantean dos alternativas que consideran 
igualmente plausibles: o bien ADd representa un mecanismo psicológico no social que se exacerba en contextos sociales; o bien representa un mecanismo social que puede en ocasiones ser aplicado equivocadamente en contextos no sociales. La alternativa favorecida por los autores en este estudio se orientaba en esta última línea: ADd es el resultado de un mecanismo más amplio, que opera en contextos sociales, en efecto, pero que no necesariamente evolucionó para eso (es decir, no fue seleccionado por esa función específica), a saber, la aversión a recibir una recompensa menor a la esperada: cada vez que (por las razones que sea: correctas o incorrectas, delirantes o atendibles) esperamos una recompensa determinada y recibimos, en cambio, una recompensa menor, experimentamos una sensación de frustración que nos lleva a adoptar una actitud negativa ante dicha recompensa, la cual puede exteriorizarse de dos maneras (cuanto menos): expresando nuestra desaprobación, o rechazando la recompensa. Las ventajas de este modelo de explicación son varias: en primer lugar, nos permite pensar (al menos algunas de) nuestras prácticas judicativas en un contexto más amplio que el contexto social, dado que la aversión a recibir una recompensa menor a la esperada puede operar aun en contextos no sociales. La segunda ventaja de la explicación a partir de la "recompensa esperada" es que presupone operaciones cognitivas (relativamente) simples, dado que no implica que el sujeto posea, por ejemplo, una teoría de la mente o que realice atribuciones de intencionalidad. En consecuencia, y ésta es la tercera ventaja, ADd no se vuelve necesariamente privativa de especies animales dotadas de capacidades cognitivas sofisticadas, y su presencia se vuelve posible en especies de animales no humanos, tanto dentro del orden de los primates como fuera de él.

Como advirtieron los propios autores, sin embargo, este modelo no explica por qué los índices de rechazo fueron superiores cuando el beneficiado no era un lugar vacío sino otro niño, y es en función de ello que el mismo grupo de investigación condujo, ese mismo año, un nuevo estudio (McAuliffe et al., 2013) destinado a determinar si detrás 
del rechazo de las ofertas desiguales se hallaba una mera sensación de frustración, o si además operaba, al menos en algunos casos, una sensación de resentimiento (spite). De acuerdo con los autores, el hecho de que en el nuevo estudio los niños rechazaran ofertas desiguales aun en los casos en los que dicho rechazo no alteraba en lo más mínimo la recompensa recibida, solo puede ser explicado suponiendo que, a diferencia de otras especies animales como los chimpancés, en el caso de los seres humanos ADd puede estar expresando un deseo inconsciente de perjudicar al otro a fin de impedirle adquirir una ventaja relativa respecto de uno mismo. Desde esta perspectiva, ADd se vuelve un fenómeno primordialmente social, en la medida en que incorpora al otro en la consideración del entorno: mi bienestar no se define entonces únicamente por el hecho de que yo obtenga lo que espero, sino también por comparación con lo que recibe el otro, bajo el supuesto de que una mayor recompensa para el otro implica una desventaja para mí. La variable del resentimiento trae a la luz el hecho de que, desde esta visión agonística de la realidad social, mi consideración acerca de lo justo o injusto de una situación ya no depende de (solamente) de lo que yo no recibo, sino (también) de lo que él otro recibe y yo no, y esta actitud, presumiblemente exclusiva de nuestra especie, podría explicar gran parte de la dinámica social.

Las ventajas que señalé más arriba como propias de la explicación no social de ADd que defendían los autores en los dos primeros estudios, desde ya, se ven anuladas por este cambio de modelo de explicación: si ADd no es la expresión de un mecanismo no social, sino que es en sí mismo producto del resentimiento o de una concepción agonística de la interacción social, entonces debemos explicar qué es lo que motiva, en los experimentos, los rechazos de ofertas desventajosas cuando el otro lugar es una mera silla vacía. El principal problema, sin embargo, se vincula con el hecho de que la explicación social de ADd corta todo vínculo con otras especies, en la medida en que presupone requisitos cognitivos mucho más altos que el modelo de 
explicación alternativo.

Antes de juzgar las virtudes hermenéuticas del modelo global de Blake y McAuliffe, no obstante, debemos evaluar lo que dichos estudios revelan acerca de la contraparte de ADd, a saber, la Aversión a la Desigualdad Ventajosa [ADv].

\section{ADV: cuando la igualdad nos beneficia}

El diseño del primer estudio de Blake y McAuliffe presentaba una segunda arista decisiva para comprender el surgimiento de la "aversión a la desigualdad" evidenciada por los participantes: a lo largo del experimento, el experimentador variaba la cantidad de caramelos asignados en forma desigual a los participantes, pero esa desigualdad, en ciertas ocasiones, favorecía al participante activo en lugar de perjudicarlo, asignándole, por ejemplo, cinco caramelos a él y uno al otro participante. Ahora bien, si los rechazos de ofertas desiguales señalados más arriba fueran la expresión de una aversión global a la desigualdad, los índices de rechazo de la distribución desigual ventajosa deberían ser al menos tan altos como los de las distribuciones ventajosas para el individuo. El final del cuento, es, por supuesto, perfectamente predecible: ninguno de los niños que componían el grupo de cuatro años del estudio inicial rechazó una distribución desigual beneficiosa, aun cuando la distribución fuera marcadamente despareja (cuatro a uno). No hay nada particularmente contra-intuitivo, desde ya, en el hecho de que los niños evidencien una clara Aversión a la Desigualdad Desventajosa, pero no su contraparte, la Aversión a la Desigualdad Ventajosa: esperar que renuncie a una recompensa que lo beneficia en nombre de una norma igualitaria parece fuera del rango de posibilidades de un niño de cuatro años, y los resultados del estudio sugieren que, en efecto, la existencia de desigualdades no provoca, al menos en ese momento de la vida, ninguna sensación de incomodidad - siempre y cuando dicha desigualdad no los perjudique.

Sin embargo, los resultados globales del estudio muestran un panorama mucho más 
complejo: si bien el índice de rechazos de ofertas ventajosas se mantiene cercano a cero para todos los grupos que van desde los cuatro a los siete años, dicho índice trepa súbitamente hasta casi un $50 \%$ en el en el grupo de niños de ocho años. En otras palabras: en casi la mitad de las ocasiones en las que a un niño de ocho años se le presentaba una oferta desigual que lo beneficiaba, el sujeto prefería renunciar a su recompensa. Ahora bien, ¿a qué se debe esta variación y qué factores pueden contribuir a explicar su surgimiento (o consolidación) al llegar a los ocho años de edad?

Los autores del estudio evalúan dos posibles modelos de explicación, para luego volcarse por una tercera alternativa. La primera hipótesis evaluada consiste en suponer que las variaciones constatadas son el resultado de un mayor desarrollo, al acercarse a los 8 años, de la capacidad de auto-control por parte de los niños: de acuerdo a dicha explicación, aun los niños de cuatro años perciben la injusticia de aceptar una oferta que perjudica al otro niño, pero carecen de la capacidad de autocontrol necesaria para rechazar la oferta - capacidad que recién se desarrollaría alrededor de los 8 años. La razón principal para desestimar esta explicación es atendible: ninguno de los niños de cuatro años experimentó problemas al momento de rechazar una oferta que lo perjudicaba, lo cual es evidencia suficiente de que la capacidad de auto-control ya se halla desarrollada a esa edad. La segunda alternativa evaluada y descartada consiste en explicar las variaciones súbitas en los índices de rechazo de las ofertas ventajosas a partir de la dinámica de la reputación: de acuerdo a dicha hipótesis, la razón por la cual los niños comenzarían, al llegar a los ocho años, a rechazar las ofertas que los benefician es que es precisamente alrededor de esa época cuando su propia reputación (i.e., la imagen que los demás tienen de él) comienza a ser un motivo de reputación, y el rechazo de las ofertas injustas tendría por objetivo mejorar dicha reputación o, cuanto menos, no dañarla. Pero si esto fuera así, señalan los autores, cabría esperar que los mismos niños evidencien una 
tendencia a aceptar las ofertas que los perjudican, y los resultados muestran precisamente lo contrario: como veíamos anteriormente, los índices de rechazo de las ofertas desventajosas aumentan progresivamente, en lugar de disminuir. Blake y McAuliffe abogan, en cambio, por una explicación doble para explicar la asimetría en el desarrollo de los dos tipos de Aversión a la Desigualdad. La Aversión a la Desigualdad Desventajosa puede ser cómodamente explicada por razones evolutivas. Desde la hipótesis no social defendida en los dos primeros estudios, en efecto, la aversión a aceptar pasivamente recompensas menores a las alternativas disponibles es claramente adaptativa; desde la hipótesis social del tercer estudio, lo que es bueno para mi contrincante es malo para mí. La Aversión a la Desigualdad Ventajosa, por el contrario, puede ser interpretada como evidencia de que el niño ha comenzado a incorporar en forma consciente normas sociales, normas que exigen universalidad y que se aplican en las dos direcciones: desde el momento en que incorporo la norma de la justicia, ya no puedo seguir denunciando como injusta una desigualdad que me perjudica pero aceptarla calladamente cuando me beneficia. En otras palabras, solo puedo seguir reclamando por mi derecho a no recibir un trato desigual (ADd) en la medida en que admita mi obligación de comportarme en forma igualitaria yo mismo frente a los demás (ADv).

El panorama que, de esta forma, queda configurado por el modelo dual de explicación de nuestra aversión a la desigualdad es, en síntesis, el siguiente:

- A los cuatro años, los niños evidencian una Aversión a la Desigualdad Desventajosa, es decir, una tendencia a reaccionar en forma negativa ante un escenario en el cual la distribución de recursos los ha perjudicado.

- Dicha tendencia puede ser explicada de dos formas: a) como producto de un mecanismo psicológico espontáneo e inconsciente que lleva a los niños a rechazar una recompensa cuando se halla disponible una recompensa mejor; b) como producto de un mecanismo psicológico espontáneo e inconsciente que lleva a los niños a 
rechazar una recompensa cuando un contrincante ha recibido una recompensa mayor.

Recién a los ocho años, los niños comienzan a evidenciar en forma sistemática y sostenida una Aversión a la Desigualdad Ventajosa, es decir, una tendencia a reaccionar en forma negativa ante un escenario en el cual la distribución de recursos los ha beneficiado.

- Dicha tendencia puede ser explicada por el hecho de que es precisamente alrededor de esa edad cuando los niños comienzan a incorporar normas sociales en forma consciente y admiten la necesidad de que la norma de la igualdad se aplique en las dos direcciones, es decir, tanto cuando los perjudica como cuando los beneficia.

\section{Las dificultades del modelo dual}

Las conclusiones del modelo dual son claras y permiten realizar predicciones precisas que pueden ser testeadas tanto en escenarios experimentales como naturales. Pero los problemas que presenta son igual de claros. El primero (y quizás menor) de ellos se vincula con el abordaje cuasi-kohlbergiano que propone, y la idea de que el surgimiento de ADv puede ser identificado con precisión (a los ocho años) fue la primera que debió ser revisada, fundamentalmente a causa de los resultados obtenidos por el propio grupo de investigación. Blake et al. (2015) demostró, en efecto, que al proyectar el modelo experimental anterior a poblaciones distintas a la del estudio original (compuesta por niños provenientes de países occidentales, educados, industrializados, ricos y democráticos - WEIRD, de acuerdo al acrónimo inglés acuñado en el ámbito de la sociología contemporánea), los resultados variaban dramáticamente. Si bien la sugerencia de que ADd surge en forma sistemática alrededor de los cuatro años de edad recibió una nueva (y ampliada) confirmación, el análisis de los resultados obligó a admitir, en primer lugar, que en aquellas poblaciones en las que ADv se hace indudablemente manifiesta (en el presente estudio: Canadá, EEUU y Uganda), esto sucede en forma claramente gradual, y no en forma abrupta, 
como sugerían los estudios anteriores, o aún en una edad mucho más tardía que los ocho años (en el caso de México: alrededor de los trece años). La segunda conclusión a la que obligó el estudio fue que ADv puede no manifestarse en ningún momento en forma sostenida, tal como sucedió en el caso de India, Perú y Senegal, lo cual sugiere en forma contundente que ADv posee un claro carácter social o que, cuanto menos, existe una serie de condiciones socio-culturales que debe darse para que se vuelva una tendencia sistemática por parte del niño. En otras palabras: ADv es una posibilidad, no una necesidad; ciertas variables del entorno pueden contribuir a que los niños desarrollen una aversión a verse beneficiados más que sus pares, pero que ese no es el único desenlace posible, y, a diferencia de ADd, ADv puede nunca desarrollarse.

Estas modificaciones en las conclusiones generales respecto de ADv no representan, sin embargo, un problema decisivo para el modelo dual, dado que una de sus características era, precisamente, que atribuía a ADv un carácter marcadamente social. Los problemas más serios que dicho modelo provienen de otro lado: en primer lugar, la vinculación entre el resentimiento y ADd propuesta por los autores no ha sido la única alternativa hermenéutica que se ha ofrecido para explicar la relación el lugar del resentimiento en nuestro sentido de justicia, y está lejos de ser la más sólida y convincente (cf., a modo de ejemplo, Forber \& Smead, 2014, quienes sugieren pensar el resentimiento no como causa de ADD, sino, a la inversa, como un mero efecto colateral del desarrollo del nuestro sentido de justicia). En segundo lugar, como veremos enseguida, existe evidencia importante de que los niños recurren al criterio de igualdad mucho antes de los cuatro años, y que cuando lo hacen no es bajo la forma de $A D d$ ni de $A D v$. En segundo lugar, al menos dos estudios (que analizaremos más adelante) han demostrado que desde los cinco años en adelante los niños comienzan a realizar castigos costosos, lo cual implica, nuevamente, apelar a un criterio neutro de igualdad. 


\section{El modelo unitario: la aversión a la desigualdad como fenómeno global}

Los estudios llevados a cabo por el grupo de Blake y McAuliffe se concentraban exclusivamente en escenarios en donde el niño se veía o bien perjudicado o bien beneficiado por una distribución desigual. Esto no permitía, como es evidente, evaluar la reacción del niño ante una situación en la cual él no se viera afectado por la distribución desigual de recursos. ¿Qué sucedería en tal caso? Si la aversión a la desigualdad que hemos visto en los niños de cuatro años se activa (bajo la modalidad de $A D d$ ) como reacción ante el perjuicio que dicha distribución le ocasiona, ¿sería lógico que se active en una situación en la cual él fuera un mero espectador pasivo que no posee nada en juego en la distribución desigual? La evidencia recogida hasta el momento, curiosamente, parece indicar que sí. Dos estudios recientes (McAuliffe et al., 2013; Salali, Juda, \& Henrich, 2015) han sugerido, en efecto, que alrededor de los cinco años, los niños comienzan a castigar las distribuciones desiguales aún si las mismas no los perjudican ni benefician: en escenarios experimentales en los cuales podían o no intervenir ante una distribución desigual de recursos entre terceros, ambos estudios mostraron una tendencia a hacerlo que comienza a los cinco años y se vuelve estable y sistemática al acercarse a los 6 años. Si bien ambos estudios difieren considerablemente en cuanto a la interpretación que proponen de las causas que motivan este desarrollo, lo decisivo es que la tendencia en cuestión se aparta de la dicotomía ADd-ADv y parece sugerir claramente la existencia de una aversión global a la desigualdad, independiente de los beneficios o perjuicios que dicha desigualdad implica para el sujeto.

La importancia de esto es decisiva para nuestra comprensión de la aversión a la desigualdad: si los niños están dispuestos a castigar desigualdades que no los afectan, entonces ninguna de las explicaciones que vimos hasta el momento (propuestas por el modelo dual) parece poder explicar el origen de esta tendencia, dado que tanto la hipótesis de la frustración como la del resentimiento dependen en 
forma esencial del impacto que la desigualdad genera en el niño. Si anulamos la variable de ADd como reacción personal frente a una determinada situación, entonces, tenemos que encontrar una nueva forma de explicar el surgimiento de la aversión a la desigualdad.

En el mismo año en que Blake \& McAuliffe (2011) realizaron el primero de sus estudios, Schmidt \& Sommerville (2011) llevaron adelante el primero de una serie importante de estudios destinados a analizar la posible presencia del criterio de igualdad en niños mucho menores a los analizados por los estudios anteriores, lo cual fue posible mediante el recurso al método de tiempo de fijación de la mirada. Dicho método se basa en el presupuesto de que el tiempo de fijación de la mirada de un niño en un escenario determinado revela sus expectativas: cuando un niño detiene su mirada más de lo normal en una situación específica es porque sus expectativas han sido violadas, es decir, lo que él esperaba que sucediera no ha ocurrido, y lo que efectivamente ha sucedido llama su atención (Para una discusión de los fundamentos teóricos de la metodología del tiempo de fijación de la mirada, cf. Aslin, 2007; Oakes, 2010). Ahora bien: ¿cómo determinar si un niño ha detenido su mirada "más de lo normal" frente a una situación? Ante esta pregunta se abren dos diseños experimentales distintos: en el primero de ellas, se mide el tiempo que el niño dedica a mirar cada elemento de la secuencia de una situación cotidiana definida por parámetros naturales (como, por ejemplo, soltar una pelota y que la misma caiga y toque el piso), y luego se comparan dichos tiempos con los de un escenario en la cual esos parámetros han sido alterados (por ejemplo, soltar una pelota y que la misma no caiga hacia el piso). En el segundo tipo de diseño experimental, se habitúa al niño a esperar un determinado desenlace artificial (haciéndole ver en forma repetida una determinada secuencia hasta que la misma se vuelva "natural") y luego se altera algún elemento específico de la secuencia, comparando el tiempo de mirada en ambas situaciones. 
El método del tiempo de fijación de la mirada (en cualquiera de las dos modalidades que describí) puede ser aplicado, desde ya, no solo a un ser humano de cualquier edad sino, al menos en principio, a todo organismo vivo que posea órganos visuales. En lo que nos concierne, sin embargo, este método es particularmente interesante para comprender la estructura cognitiva de los niños, dado que permite analizar la reacción ante un determinado escenario aun antes de que el niño haya alcanzado el desarrollo cognitivo necesario para entender instrucciones y aun antes siquiera de que pueda dominar conceptos abstractos en forma precisa.

Desde el estudio pionero de Schmidt y Sommerville (2011), un conjunto importante de estudios ha contribuido a fortalecer la idea de que el momento en el que el niño comienza a presuponer la igualdad como desenlace naturalmente esperable en la distribución de recursos se sitúa mucho antes de las estimaciones previas, y aún antes que la edad propuesta por el modelo dual (cuatro años): de acuerdo a dichos estudios, en efecto, ese presupuesto ya se hace presente en los niños entre los 12 y 19 meses de edad (cf. Sloane, Baillargeon, \& Premack, 2012; Sommerville, Schmidt, Yun \& Burns, 2013; Burns \& Sommerville, 2014), lo cual, de acuerdo a Schmidt \& Sommerville, revela que ya a esa edad los niños "poseen los rudimentos de un sentido de la justicia (fairness) en cuanto a que esperan que los recursos sean distribuidos en forma igualitaria" (Schmidt \& Sommerville, 2011, p. 5).

Las consecuencias de esto son mucho más que un simple cambio en la edad: más allá del hecho de que estos resultados retrotraen el surgimiento de la idea de igualdad a la mitad de edad que el modelo anterior, lo decisivo es que ofrecen un panorama radicalmente distinto: mientras que el modelo dual sugería que la Aversión a la Desigualdad surgía inicialmente como una reacción del niño frente a una situación que lo perjudica, el modelo unitario se basa en la reacción del niño ante el hecho de que una determinada norma (la igualdad) se ha visto transgredida, lo cual presupone ya la existencia del criterio neutro de igualdad - es decir, la igualdad considerada en forma 
independiente de los beneficios o perjuicios para la persona que juzga. Y si esto es así, la Aversión global a la Desigualdad es tanto lógica como temporalmente anterior al surgimiento no sólo de ADv sino también de ADd. Aun cuando esto podría parecer una perogrullada, es importante recordar que el modelo dual proponía precisamente lo contrario: primero desarrollamos ADd -por distintas razones- y luego desarrollamos el concepto de igualdad - entendido en términos de complementariedad entre ADd y ADv. De acuerdo a modelo unitario, por el contrario, para cuando ADd empieza a hacerse claramente visible en las decisiones y acciones del niño, el criterio de igualdad ya se encuentra claramente desarrollado. Desde ya, (haciendo a un lado por un momento la edad propuesta por cada modelo) las predicciones de ambos son claramente distintas: de acuerdo al modelo dual, antes de alcanzar un determinado momento en su desarrollo, la única desigualdad que molesta al niño es aquella que lo perjudica directamente; de acuerdo al modelo unitario, por el contrario, el niño experimenta extrañeza ante las situaciones de desigualdad mucho antes de comenzar manifestar una aversión a la desigualdad que lo perjudica - extrañeza que proviene del hecho de que se ha violado una cierta expectativa: la expectativa de igualdad.

Cabe remarcar, sin embargo, que esta expectativa de igualdad tiene un carácter claramente social, no en cuanto a su génesis (sobre lo que volveremos más adelante), sino en cuanto al ámbito en el cual se activa espontáneamente. Podemos concluir que esto es así a partir de dos condiciones de control incorporadas en el diseño experimental de Sloane et al. (2012). La primera de ellas consistió en analizar el tiempo de fijación de la mirada de los niños ante una distribución desigual que no iba dirigida a personas sino a objetos inanimados. Los resultados fueron nulos: ninguno de los niños fijó la mirada durante un tiempo significativamente mayor en tales escenarios, lo cual habría sucedido si la expectativa de igualdad transcendiera a (o fuera independiente de) el ámbito social. La incorporación de una segunda condición de control permitió constatar, asimismo, que la expectativa de igualdad desaparecía 
por completo cuando no estaba claro para el niño si el reparto desigual había sido producto de la voluntad del experimentador, es decir: cuando los niños enfrentaban un reparto desigual pero sin saber cómo se había producido. Esto implica, como se hace evidente, que no es el resultado en sí lo que llama la atención de los niños (es decir, el mero hecho de que se haya producido un reparto desigual), sino el hecho de que dicho resultado sea producto de la decisión de otro sujeto. Si esto es así, podemos entonces que al menos desde los 12/15 meses en adelante los niños parecen reaccionar con sorpresa ante un reparto desigual, pero sólo cuando el mismo es producto de una decisión humana y se halla dirigido a un ser humano.

A modo de síntesis:

Al menos desde los 12-15 meses de edad, los niños esperan que las distribuciones de recursos sean igualitarias.

- Esta expectativa de igualdad tiene un carácter claramente social en cuanto a su ámbito de activación, dado que desaparece: a) cuando el destinatario de la distribución no es un ser humano, o b) cuando el niño desconoce el proceso mediante el cual se produjo la distribución desigual.

- La expectativa de igualdad se activa aun cuando el niño no se vea personalmente beneficiado ni perjudicado por la distribución de recursos.

- Dicha expectativa de igualdad se hace manifiesta en la proclividad de los niños, desde los cinco años de edad, a castigar las distribuciones desiguales, aun cuando las mismas no los perjudiquen ni beneficien, y están dispuestos a sacrificar parte de sus propios recursos con tal de castigar dichas distribuciones (siempre y cuando el sacrificio sea relativamente bajo).

\section{El modelo unitario y el origen de la expectativa de igualdad}

De acuerdo al modelo unitario, entonces, la expectativa de igualdad posee un carácter claramente social en cuanto a su ámbito de activación espontánea. Pero ¿qué hay 
respecto de su origen? ¿Es esta expectativa también un producto social, una hija de la cultura, como sugería el modelo dual? ¿O posee raíces más profundas y más generales?

Las respuestas que han recibido estos interrogantes por parte de los investigadores a cargo de los estudios parecen prácticamente agotar todas las posibilidades lógicas. Sommerville et al. $(2013,64)$, por ejemplo, plantean que la presencia de esta expectativa de igualdad en los niños desde una edad tan temprana podría ser el producto de un proceso de inducción realizado por el niño (en forma inconsciente) a partir de la constatación de una regularidad estadística: el niño percibe cotidianamente que los bienes son repartidos en forma igualitaria, y esto lo lleva a esperar repartos similares en lo sucesivo. Sloane et al. (2012), por su parte, consideran una alternativa similar, pero enfatizan la posible incorporación por parte del niño de esta expectativa de igualdad no como mera regularidad natural, sino como una norma, una regla. A pesar de que ambas propuestas asignan un carácter inductivo y, por lo tanto, sociocultural a la expectativa de igualdad, la diferencia entre ambas propuestas es importante, dado que la incorporación de una regularidad como norma tiende a dotarla de una dimensión moral que está ausente en la mera regularidad estadística.

Esta posible dimensión específicamente moral de la expectativa de igualdad se ve radicalizada en una tercera alternativa de explicación propuesta por este último estudio, a saber, que la expectativa de igualdad quizás forma parte de un cierto conjunto de criterios y normas que poseemos en forma innata. Desde esta perspectiva, los seres humanos estaríamos programados desde nuestro nacimiento para percibir un desenlace igualitario como normal, lo cual llevaría a que toda violación de dicha expectativa produzca una reacción negativa - reacción que puede ir desde la mera sorpresa pasiva (evidenciada por tiempos de fijación de la mirada más prolongados) hasta acciones concretas como la decisión de castigar distribuciones desiguales, aun cuando no nos beneficien o perjudiquen. 
Una tercera alternativa entre estas dos perspectivas (socio-cultural versus innata) ha sido propuesta para explicar el origen de nuestra expectativa de igualdad: en el primero de los estudios de lo que he denominado modelo unitario, Schmidt \& Sommerville (2011) encontraron una clara correlación entre la expectativa de igualdad de los niños y la actitud de los mismos en escenarios en los que se veían obligados a compartir recursos: los "altruistas" (esto es, aquellos niños que en un experimento previo habían demostrado una tendencia clara a compartir sus propios recursos con otros niños) prestaron más atención (92\%) a las distribuciones desiguales que los niños "egoístas" (62\%). Si bien sólo en forma especulativa, los autores sugieren tentativamente, en función de estos resultados, que la tendencia a actuar en forma pro-social (en este caso, a compartir los propios recursos) podría ser la causa de la expectativa de igualdad; en otras palabras, que los niños que poseen tendencias prosociales más marcadas tienden, por esa razón, a esperar una distribución igualitaria aun cuando la misma no los afecte, y, de modo inverso, que aquellos niños en quienes la tendencia pro-social es menos marcada no suelen operar bajo el presupuesto de la igualdad y, en consecuencia, no se sorprenden cuando enfrentan un escenario desigual. Esta no es, por supuesto, la única forma de explicar los resultados obtenidos por el estudio (lo cual explica que los autores presenten su interpretación como puramente especulativa), y al menos dos explicaciones alternativas son igualmente plausibles: a) que ambas tendencias (la tendencia a compartir y la expectativa de igualdad) sean producto de un tercer mecanismo psicológico previo a las mismas; b) que la tendencia a compartir sea el efecto ( $y$ no la causa) de la expectativa de igualdad.

Como señalaba al inicio de la sección, las alternativas reseñadas parecen prácticamente agotar todas las alternativas de respuesta al interrogante acerca del origen de nuestra expectativa de igualdad, y esta clara ausencia de consenso contrasta marcadamente con los resultados que han arrojado los estudios hasta el 
momento. En efecto, si tomamos en consideración la totalidad de los resultados obtenidos a partir de los experimentos tanto del modelo dual como del modelo unitario, creo que el panorama que emerge ante nuestros ojos se vuelve relativamente preciso, y podría ser resumido como sigue:

Al menos desde los 12-15 meses de edad, los niños esperan que las distribuciones de recursos sean cuantitativamente igualitarias.

- Esta expectativa de igualdad tiene un carácter claramente social en cuanto a su ámbito de activación, y se activa aun cuando el niño no se vea personalmente beneficiado ni perjudicado por la distribución de recursos.

- Las manifestaciones de dicha expectativa de igualdad varían desde una actitud de sorpresa pasiva hasta una clara aversión a la desigualdad, la cual comienza a hacerse manifiesta: a) alrededor de los cuatro años en escenarios en los que el niño se ve personalmente perjudicado (ADd); b) alrededor de los cinco años en escenarios en los que está dispuesto a sacrificar recursos propios para castigar repartos desiguales ("castigo costoso de terceros", o "castigo altruista"); c) desde los siete u ocho años en adelante en escenarios en los que el niño se ve personalmente beneficiado (ADv).

Desde ya, como hemos visto, la explicación que cada modelo propone de los procesos que fundamentan cada uno de estos momentos es radicalmente distinta. Los resultados de los experimentos de ambos modelos son, sin embargo, conciliables y presentan un esquema de desarrollo sumamente consistente, articulado alrededor de la idea de una expectativa de igualdad presente en los niños desde al menos los dos años de edad.

Claro está que la mera presencia de esta expectativa de igualdad no garantiza decisiones y acciones igualitarias. Que el niño opere (por las razones que sea) bajo el presupuesto de que los repartos de recursos deberían ser igualitarios no implica en absoluto que el criterio de la igualdad vaya a sea la guía efectiva de sus acciones. En escenarios experimentales, esto se hace particularmente evidente en dos estudios 
complementarios realizados por el grupo de P. Blake (Blake, McAuliffe, \& Warneken, 2014; Smith, Blake, \& Harris, 2013), los cuales abordaron el objetivo de comprender la brecha conocimiento-acción, es decir, el desfasaje entre saber que uno debe hacer algo y efectivamente hacerlo. En los estudios mencionados, los niños analizados declaraban en forma explícita que, frente a un reparto específico de recursos determinado por el experimento, lo correcto sería distribuirlos en forma igualitaria, pero simultáneamente predecían que, ante una situación de ese tipo, probablemente ellos no se atendrían al criterio de igualdad, sino que optarían por beneficiarse a sí mismos. Una explicación frecuente para este tipo de desfasaje es asumir que se trata de un caso de debilidad de la voluntad: estoy convencido de que debo hacerlo, pero mi voluntad es débil. Como resaltan los autores, sin embargo, ése no parece ser el caso en absoluto en el escenario analizado, dado que los niños predicen en forma explícita que ellos optarían por un reparto egoísta antes que por uno igualitario, aun sabiendo que éste último representa la opción moralmente correcta. La explicación que los autores propone, en cambio, es que de lo que se trata en este caso es un conflicto entre, por un lado, la convicción (consciente) de que debo ser igualitario, y, por otro, el deseo (inconsciente) de preservar mi ventaja relativa frente al contrincante.

Esta explicación, sumamente plausible en sí misma, se limita a tomar en cuenta una sola de las variables que pueden obstaculizar el pasaje de la creencia a la acción. Como han mostrado Burns \& Sommerville (2014) el criterio de la igualdad puede también verse claramente derrotado frente al de la pertenencia a un grupo: tomando como objeto de análisis las reacciones de un grupo de niños de 15 meses de edad, los autores constataron que la tendencia a preferir los repartos igualitarios se veía mayoritariamente suspendida cuando se incorporaba la variable de la raza a la que pertenecían los experimentadores: en un escenario de reparto de recursos a terceros, los niños (de raza caucásica) tendían a considerar injusta una distribución desigual de recursos solo si los dos sujetos que recibían la distribución pertenecían a dicha raza, 
pero no la consideraban injusta cuando el sujeto perjudicado por la distribución pertenecía a otra raza.

Independiente de las proyecciones (sumamente preocupantes) de este último experimento, lo decisivo es que contribuye a mostrar que la presencia de la expectativa de igualdad no garantiza decisiones igualitarias, y que esto se debe a que las consideraciones que pueden intervenir son mucho más numerosas que el deseo de preservar la ventaja relativa - lo cual refuerza la necesidad de distinguir claramente, como he propuesto, entre el sentido de justicia en sí mismo y los criterios sobre los que el mismo opera, entre los cuales se cuentan, además de la igualdad, el mérito y la reciprocidad, y es a ellos a los que nos dedicaremos en las secciones siguientes.

\section{Conclusiones}

¿Qué sabemos, al menos hasta el momento, respecto de la ontogénesis de nuestro sentido de justicia o, más bien, de los criterios sobre los que nuestro sentido de justicia se apoya para evaluar nuestras interacciones con el entorno?

Respecto del criterio del criterio de reciprocidad, sabemos que es de los primeros en hacerse manifiesto en las evaluaciones de los niños: entendido en términos de reciprocidad actitudinal, la evidencia sugiere que, al momento de decidir otorgarle o no un beneficio a otro sujeto, los niños comienzan ya desde el primer año de edad a verse influenciados por los intercambios que han tenido previamente con dicho sujeto. Esto no implica, desde ya, que al hacer esto los niños estén realizando cálculos mentales precisos que involucren cuantificación u conversiones de equivalencia: apoyándonos en el concepto de reciprocidad actitudinal, podemos suponer que de lo que se trata es simplemente del hecho de que las interacciones previas han producido en él una predisposición afectiva específica (positiva o negativa) que influencia su decisión. La reciprocidad calculada parece hacerse manifiesta bastante más tarde (no 
antes de los tres años y medio, de acuerdo a los cálculos más optimistas), y la reciprocidad potencial recién al alcanzar los cinco años - momento en el cual las acciones de los niños comienzan a involucrar una racionalidad estratégica que evalúa los beneficios que se derivarían de beneficiar a un sujeto con quien pueden volver a encontrarse en un escenario de intercambio. (Cf. House, Henrich, Sarnecka, \& Silk, 2013; Olson \& Spelke, 2008; Sebastián-Enesco \& Warneken, 2015; Warneken \& Tomasello, 2013).

En cuanto al criterio de igualdad, la evidencia que hemos relevado en las páginas previas sugiere que el mismo comienza a manifestarse alrededor de la misma época: entre los 12 y los 15 meses de edad, los niños esperan que las distribuciones de recursos sean igualitarias, aun en escenarios en los que no están personalmente involucrados. Alrededor de los 4 años de edad, la expectativa de igualdad comienza a traducirse en acciones en forma sistemática, y a los cinco años comienza a traduce en la disposición a castigar las distribuciones desiguales, aun si esta distribución no los afecta ("castigo de terceros" o "castigo altruista"), y aun si para poder llevar a cabo ese castigo deben invertir recursos propios ("castigo costoso"). Finalmente, recién al llegar a los siete u ocho años, y dependiendo de ciertas condiciones socio-culturales, la expectativa de igualdad se expresa en rechazos explícitos de desigualdades que benefician al propio sujeto.

El criterio de mérito, por su parte, parece entrar en escena con algo de posterioridad respecto de los otros dos, dado que la evidencia sugiere que no es hasta los tres años de edad que las evaluaciones de los niños comienzan a operar sobre la expectativa de equidad, es decir, la expectativa de que los recursos la distribución de recursos sea acorde con la contribución realizada por cada uno de los participantes en una tarea cooperativa - una expectativa que se halla ausente en contextos colectivos no cooperativos, pero que se manifiesta aun cuando el niño no se halle personalmente involucrado en la tarea. Por último, el hecho de que las evaluaciones de mérito de los 
niños se apoyen a una edad tan temprana en cierto cálculo respecto de la proporción en la que cada uno ha contribuido, sugiere que los cálculos de mérito pueden ser independientes de la capacidad lógica de realizar cálculos de proporción en forma abstracta y explícita - dado que dicha capacidad solo se adquiere mucho después. (Cf. Blake, McAuliffe, \& Warneken, 2014; Hamann, Bender, \& Tomasello, 2014; Hamann, Warneken, Greenberg, \& Tomasello, 2011; Kanngiesser \& Warneken, 2012; Liénard, Chevallier, Mascaro, Kiura, \& Baumard, 2013; Ng, Heyman, \& Barner, 2011; Sloane et al., 2012; Warneken, Lohse, Melis, \& Tomasello, 2011).

Sabemos, en síntesis, en qué momento aproximado de su desarrollo es posible que un niño comience a recurrir a ciertos criterios, y sabemos también cuáles son algunas de las condiciones bajo las cuales esto probablemente sucederá. Todo este conocimiento, sin embargo, es claramente limitado y tentativo: limitado porque, como siempre, lo que sabemos (o creemos saber) es mucho menos que lo que no sabemos; tentativo porque bien podría suceder que todas las investigaciones realizadas hasta el momento sean puestas en cuestión o revisadas sustancialmente con el correr de los años por estudios posteriores. En cuanto a lo que no sabemos, cabe resaltar adicionalmente el hecho de que hasta el momento solo tres criterios de justicia han sido estudiados en el marco de la psicología del desarrollo (igualdad, mérito y reciprocidad), pero es lógicamente posible (y completamente probable) que nuestro sentido de justicia apele en realidad a un conjunto mucho más amplio de criterios al evaluar nuestras interacciones con el entorno: los criterios de propiedad y territorialidad, por ejemplo, que sí han comenzado a ser estudiados en otras especies, no han sido abordados hasta el momento (al menos hasta donde sabemos) por la psicología del desarrollo, y el papel que ambos criterios juegan en nuestras evaluaciones permanece una incógnita. Aún así, los progresos realizados a lo largo de la última década han sido inmensos, las bases para nuevas investigaciones se hallan claramente asentadas, y los resultados obtenidos permiten extraer algunas conclusiones generales, 
fuertemente especulativas, por supuesto, pero apoyadas al menos sobre cierta base empírica.

Cabe abordar, antes de concluir, una objeción potencial: ¿son verdaderamente relevantes las investigaciones llevadas a cabo por la psicología del desarrollo para comprender nuestro sentido de justicia? Ciertamente, los escenarios que hemos visto analizados por los investigadores (la mayor parte de ellos artificiales) parecen ridículamente simples e irrelevantes para comprender fenómenos sociales complejos, dado que se limitan a abordar la reacción y expectativas de los niños ante una distribución de recursos materiales, lo cual parece hallarse a una distancia enorme del tipo de problemáticas que suelen interesarnos cuando abordamos el problema de la justicia. Pero que esto no es así se hace evidente si admitimos, como creo que debemos necesariamente hacerlo, que los mecanismos psicológicos sobre los cuales se asientan las reacciones de los niños son los mismos mecanismos sobre los que se van a asentar los fenómenos más complejos, tales como los reclamos de derechos de una minoría, los reclamos gremiales de salarios diferenciados de acuerdo al riesgo y la complejidad de cada tipo de labor desarrollada, o los reclamos relativos a la pena de muerte. Considerados desde esta perspectiva, las manifestaciones más básicas de las expectativas de igualdad (o de mérito y reciprocidad) que vemos surgir desde el primer año de edad representan los tornillos y engranajes más básicos de nuestro increíblemente complejo sentido de justicia. Cabe remarcar, desde ya, que investigaciones futuras bien podrían impugnar la totalidad de los resultados reseñados y mostrar que los mismos no son más que un fenómeno histórico y cultural, producto, por ejemplo, de las condiciones socio-económicas sobre las que se asienta el capitalismo moderno. Una impugnación de ese tipo, sin embargo, debería, en primer lugar, proceder por la vía experimental, en lugar de simplemente dar por supuesto el carácter estrictamente cultural de nuestro sentido de justicia, y, en segundo lugar, poder explicar la evidencia que parecería sugerir que dichos mecanismos psicológicos 
se hallan presentes en numerosas especies de primates no humanos (cf., al respecto, Braicovich 2019). No hay ningún obstáculo lógico que haga imposible que ambos objetivos sean alcanzados, pero representan, en todo caso, tareas todavía pendientes.

\section{Referencias}

Aslin, R. N. (2007). What's in a look?. Developmental Science, 10(1), 48-53.

Blake, P. R., \& McAuliffe, K. (2011). "I had so much it didn't seem fair”: Eight-year-olds reject two forms of inequity. Cognition, 120(2), 215-224.

Blake, P. R., McAuliffe, K., Corbit, J., Callaghan, T. C., Barry, O., Bowie, A., ... Warneken, F. (2015). The ontogeny of fairness in seven societies. Nature, 528(7581), 258-261.

Blake, P. R., McAuliffe, K., \& Warneken, F. (2014). The developmental origins of fairness: The knowledge-behavior gap. Trends in Cognitive Sciences, 18(11), $559-561$

Braicovich, R. (2019). Hacia una filogénesis del sentido de justicia. Una revisión de la evidencia proveniente de la primatología. Aceptado para publicación.

Burns, M. P., \& Sommerville, J. A. (2014). "I pick you": the impact of fairness and race on infants' selection of social partners. Frontiers in Psychology, 5.

Forber, P., \& Smead, R. (2014). The evolution of fairness through spite. Proceedings of the Royal Society of London B: Biological Sciences, 281(1780), 2013-2439.

Hamann, K., Bender, J., \& Tomasello, M. (2014). Meritocratic sharing is based on collaboration in 3-year-olds. Developmental Psychology, 50(1), 121-128.

Hamann, K., Warneken, F., Greenberg, J. R., \& Tomasello, M. (2011). Collaboration encourages equal sharing in children but not in chimpanzees. Nature, 476(7360), 328-331.

House, B., Henrich, J., Sarnecka, B., \& Silk, J. B. (2013). The development of contingent reciprocity in children. Evolution and Human Behavior, 34(2), 86-93. 
Kanngiesser, P., \& Warneken, F. (2012). Young children consider merit when sharing resources with others. PLOS ONE, 7(8).

Kohlberg, L. (2003). Psicología del desarrollo moral. Bilbao: Desclée de Brouwer.

Liénard, P., Chevallier, C., Mascaro, O., Kiura, P., \& Baumard, N. (2013). Early understanding of merit in Turkana children. Journal of Cognition and Culture, 13(1-2), 57-66.

McAuliffe, K., Blake, P. R., Kim, G., Wrangham, R. W., \& Warneken, F. (2013). Social influences on inequity aversion in children. PLOS ONE, 8(12), e80966.

Ng, R., Heyman, G. D., \& Barner, D. (2011). Collaboration promotes proportional reasoning about resource distribution in young children. Developmental Psychology, 47(5), 1230-1238.

Oakes, L. M. (2010). Using habituation of looking time to assess mental processes in infancy. Journal of Cognition and Development, 11(3), 255-268.

Olson, K. R., \& Spelke, E. S. (2008). Foundations of cooperation in young children. Cognition, 108(1), 222-231.

Salali, G. D., Juda, M., \& Henrich, J. (2015). Transmission and development of costly punishment in children. Evolution and Human Behavior, 36(2), 86-94.

Schmidt, M. F. H., \& Sommerville, J. A. (2011). Fairness expectations and altruistic sharing in 15-month-old human infants. PLOS ONE, 6(10), e23223.

Sebastián-Enesco, C., \& Warneken, F. (2015). The shadow of the future: 5-year-olds, but not 3-year-olds, adjust their sharing in anticipation of reciprocation. Journal of Experimental Child Psychology, 129, 40-54.

Sloane, S., Baillargeon, R., \& Premack, D. (2012). Do infants have a sense of fairness? Psychological Science, 23(2), 196-204.

Smith, C. E., Blake, P. R., \& Harris, P. L. (2013). I should but I won't: Why young children endorse norms of fair sharing but do not follow them. PLOS ONE, 8(3), e59510. 
Sommerville, J. A., Schmidt, M. F. H., Yun, J., \& Burns, M. (2013). The development of fairness expectations and prosocial behavior in the second year of life. Infancy, 18(1), 40-66.

Warneken, F., Lohse, K., Melis, A. P., \& Tomasello, M. (2011). Young children share the spoils after collaboration. Psychological Science, 22(2), 267-273.

Warneken, F., \& Tomasello, M. (2013). The emergence of contingent reciprocity in young children. Journal of Experimental Child Psychology, 116(2), 338-350.

\section{Declaración de contribución de los autores}

RB contribuyó en la totalidad del artículo.

\section{Editor de sección}

El editor de sección de este artículo fue Javier Romano.

ORCID ID: 0000-0002-5594-9104

\section{Formato de citación}

Braicovich, R. (2020). Ontogénesis del sentido de justicia. El criterio de igualdad analizado desde la psicología del desarrollo. Psicología, Conocimiento y Sociedad, 10(2), 150-180. doi: http://dx.doi.org/10.26864/PCS.v10.n2.7 